\title{
Fault Detection by Means of Hilbert-Huang Transform of the Stator Current in a PMSM With Demagnetization
}

\author{
Antonio Garcia Espinosa, Javier A. Rosero, Jordi Cusidó, Luis Romeral, and Juan Antonio Ortega
}

\begin{abstract}
This paper presents a novel method to diagnose demagnetization in permanent-magnet synchronous motor (PMSM). Simulations have been performed by 2 -D finite-element analysis in order to determine the current spectrum and the magnetic flux distribution due to this failure. The diagnostic just based on motor current signature analysis can be confused by eccentricity failure because the harmonic content is the same. Moreover, it can only be applied under stationary conditions. In order to overcome these drawbacks, a novel method is used based upon the Hilbert-Huang transform. It represents time-dependent series in a 2-D time-frequency domain by extracting instantaneous frequency components through an empirical-mode decomposition process. This tool is applied by running the motor under nonstationary conditions of velocity. The experimental results show the reliability and feasibility of the methodology in order to diagnose the demagnetization of a PMSM.
\end{abstract}

Index Terms-Demagnetization, empirical-mode decomposition (EMD), fault diagnosis, finite-element analysis (FEA), HilbertHuang transform (HHT), permanent-magnet synchronous motor (PMSM).

\section{INTRODUCTION}

$\mathbf{P}$ ERMANENT-MAGNET synchronous-motor (PMSM) machines are attractive for a variety of applications, such as aerospace and automotive drives, because of their high-power density, wide constant-power speed range, and excellent efficiency [1], [2]. Thus, more PMSM machines are being used in critical high-performance applications. It is for this reason that the early detection and fault diagnosis are acquiring great importance.

Several studies have been carried out in fault detection of permanent-magnet machines with different degree of succeed due to the utilized tool [3]. In this context, new tools have been investigated and implemented in the technical literature, in order to overcome the previous drawbacks and improve the obtained results [4]-[10]. The finite-element analysis (FEA), allowing

Manuscript received April 3, 2008; accepted November 24, 2009. Date of publication February 2, 2010; date of current version May 21, 2010. This work was supported by the economic support from the Spanish Ministry of Science and Technology under the DPI 2004-03180 Research Project. The work of J. A. Rosero was supported by the Program Alban, the European Union Programmed of High Level Scholarships for Latin America, under Scholarship E04D027632CO. Paper no. TEC-00127-2008.

The authors are with the Motion Control and Industrial Applications Group, Universitat Politècnica de Catalunya, TR 2, 08222 Terrassa, Spain (e-mail: garciae@ee.upc.edu; rosero@eel.upc.edu; jcusido@eel.upc.edu; romeral@eel.upc.edu; ortegar@eel.upc.edu).

Color versions of one or more of the figures in this paper are available online at http://ieeexplore.iee.org.

Digital Object Identifier 10.1109/TEC.2009.2037922 the coupling between the nonlinear magnetic and electric circuits, is taken into account for the motor analysis to predict the performance characteristics of a system, especially when the system is under fault conditions [11].

The fast Fourier transform (FFT) of the stator current has been applied to detect demagnetization faults by analyzing specific harmonics. However, it cannot be applied to nonstationary signals. Moreover, FFT analysis can not differentiate harmonics due to demagnetization from others due to eccentricity [1], [12].

Time-frequency analysis methods have been used for nonstationary signal feature extraction, although successful application of these techniques requires understanding of their respective limitations. The selection of a suitable window size is required when applying the short-time Fourier transform (STFT) to match with the specific frequency content of the signal, which is generally not known a priori. Moreover, there is a limitation between time and frequency limitations. A very appealing feature of the continuous wavelet transform (CWT) is that it provides a uniform resolution for all the scales [13]. Limited by the size of the basic wavelet function, the downside of the uniform resolution is a uniformly poor resolution. Moreover, an important limitation of the wavelet analysis is its nonadaptive nature. Once the basic wavelet is selected, it is used to analyze the whole frequency range [14]. A basic time-frequency representation is done by the Wigner-Ville distribution (WVD), which is a part of the Cohen class of distribution [15]. The difficulty with this method is the severe cross terms as indicated by the existence of negative power for some frequency ranges. In addition, the WVD of discrete time signals suffers from the aliasing problem.

The Hilbert-Huang transform (HHT) is based on the instantaneous frequencies resulting from the intrinsic-mode functions (IMFs) of the signal being analyzed [16]; thus, it is not constrained by the uncertainty limitations with respect to the time and frequency resolutions to which other time-frequency techniques are subject. In recent years, HHT has been applied to transient signal analysis and bearing identification of damage [17], [18].

In this paper, the effects of flux disturbances due to chipped or locally demagnetized magnets in PMSM machines are researched through simulations and experiments.

First, in Section II, simulations have been carried out by means of 2-D FEA for different velocities and demagnetizations conditions. Currents and flux density are presented, and their harmonic content is obtained. The purpose of the simulations is to find out the appearing fault frequencies, or at least to determine a range. In Section III, the novel approach for 


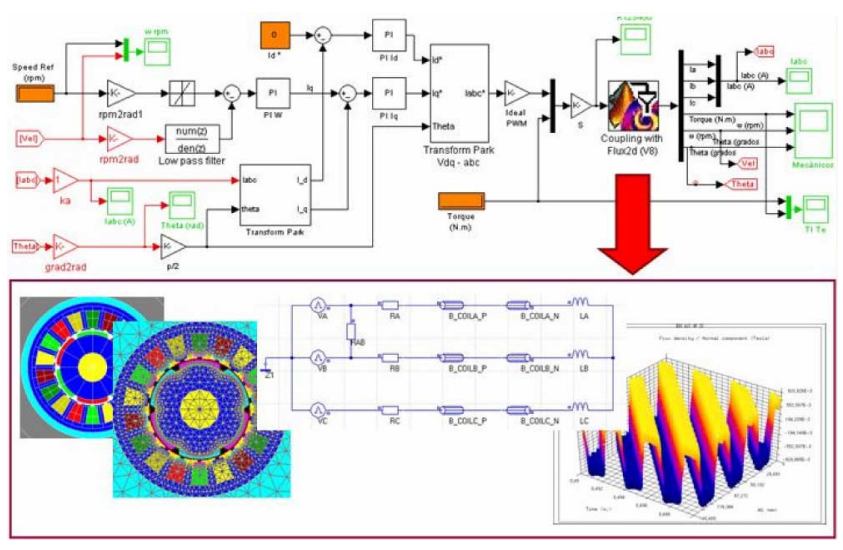

Fig. 1. Schematic of the motor and electronics model for a PMSM.

nonlinear, nonstationary data analysis, the HHT is presented for better understanding. Then, this methodology is performed to the different acquired current spectrums in order to validate this novel proposed tool. Finally, in Section V, it is concluded that this method can effectively diagnoses demagnetization failure of the PMSMs under nonstationary conditions.

\section{Simulation of PMSM With Demagnetization PERMANENT MAGNET}

Analysis and development of fault detection methods needs, as a previous stage, good knowledge of the motor behavior under fault conditions. Electrical variables such as currents and fluxes are the signals to be considered.

Compact parametric models of the motor are usual tools if the motor is considered to be in healthy state, i.e., to obtain the torque-speed ratio, to analyze and develop control algorithms, etc. On the other hand, faulty parametric models of the motor are used to acquire electromechanical faulty signals, which are represented, in general, as time-evolution signals or specific harmonics due to faults if stationery conditions can be assumed. However, parametric models assume symmetry in mechanical and electromagnetic fields, and this symmetry is missing in case of fault. For this reason, too many complex parametric models result if we attempt to model a full representation of every mechanical, electrical, or magnetic part of the motor. To overcome this drawback, simulations by means of FEA can be carried out.

Numerical simulations were developed over a model obtained with the combination of a finite-element software, Flux2D [19] for the motor model, and MATLAB-Simulink for electronics and control. Both circuits, electromagnetic and electric, have been coupled automatically by linking local variations in flux with the circuit voltage, as shows in Fig. 1.

\section{A. Partial Demagnetization of Rotor Magnet}

The permanent magnets of a PMSM can be demagnetized by high stator currents. This demagnetization phenomenon is mainly due to armature reaction especially where strong starting torque is required. Other reasons may include high short-circuit currents produced by inverter or stator faults, loads, varying

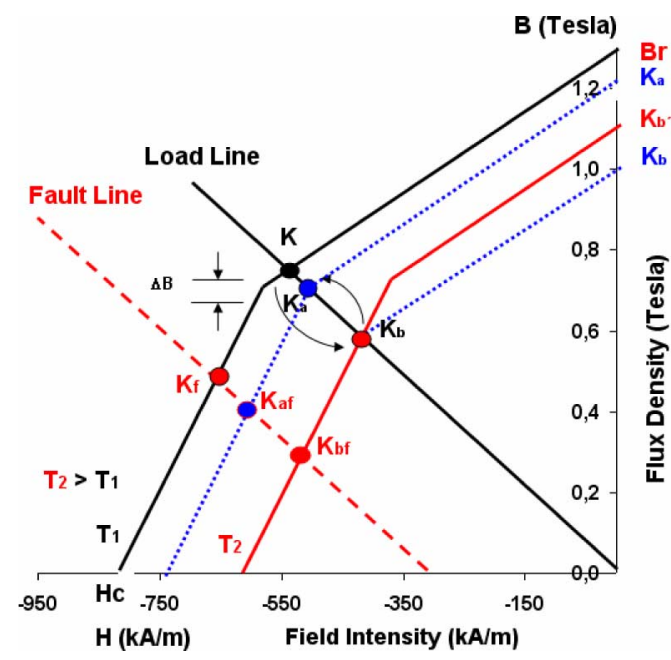

Fig. 2. Demagnetization curve of a permanent magnet (PM).

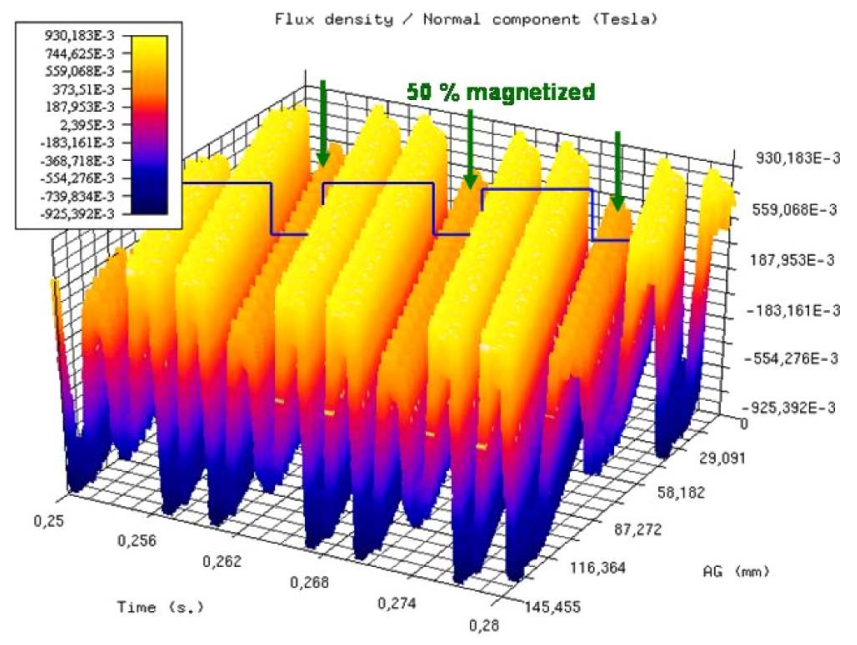

Fig. 3. Flux density distribution in air gap of a PMSM with 50\% magnetized.

working temperatures, and the own ageing of the magnet. Moreover, a short circuit of the winding can be troublesome because of drag torque and potential overheating of the motor [1]. The demagnetization can provoke irreversible losses that cause the operating point to "fall off" at the lower end of a recoil line, and then appearing as an irreversible flux loss [20], as shows in the Fig. 2. The permanent magnets can be demagnetized by fault currents such as short-circuit currents produced by inverter or stator faults, with an overrunning load, or where two motors are coupled to a single load. Moreover, short circuit of the winding can be troublesome because of drag torque and potential overheating of the motor [12].

As a consequence of demagnetization, the distributed magnetomotive force (MMF) is not sinusoidal. Thus, the amplitude of MMF has constant amplitude except under the pair of poles where the fault occurs (see Fig. 3).

So, the MMF under failure, together with the constant permeance, induces currents of main multiple frequency. If demagnetization exists, low-frequency components in the current near 
the fundamental appear [21], given by

$f_{\mathrm{dmg}}=f_{s}\left(1 \pm \frac{k \pm 1}{p}\right), \quad f_{s}=p f_{r}, \quad k=1,2,3, \ldots$

In case of constant speed at high and medium speeds, FFT allows detecting demagnetization fault by analyzing only the speed, and also it is not possible to apply FFT when there are speed and torque changes. In such a way, a new processing tool is presented next, so that it can be applied in the aforesaid cases.

\section{HTT ANALYSIS}

Each of the traditional time-frequency analysis techniques have their own limitations [22], [23]. The consequence is the misleading energy-frequency distribution for nonlinear and nonstationary data. The principle of the HTT is based on the physical time scales that characterize the oscillations of the phenomena. The local energy and the instantaneous frequency derived from the IMFs through the Hilbert transform can give us a full energy-frequency-time distribution of the data [23]; it would be the ideal tool for nonlinear and nonstationary data analysis.

\section{A. Empirical-Mode Decomposition}

An IMF is a function that satisfies two conditions. The first, in the whole dataset, the number of extreme and the number of zero crossings must either be equal or differ at most by one, and the second, at any point, the mean value of the envelope defined by the local maxima and the envelope defined by the local minima is zero.

The EMD extracts the first IMF by the following sifting process [22].

1) Find the upper envelope of $x(t)$ as the cubic spline interpolated of its local maxima, and the lower envelope, as the cubic spline interpolated of its local minimum.

2) Compute the envelope mean $m(t)$ as the average of the upper and lower envelopes.

3) Compute $h(t)=x(t)-m(t)$.

4) If the sifting result $h(t)$ is an IMF, stop. Otherwise, treat $h(t)$ as the signal and iterate on $h(t)$ through steps $1-4$. The stopping condition is

$$
\sum_{t} \frac{\left[h_{k-1}(t)-h_{k}(t)\right]^{2}}{h_{k-1}^{2}(t)}<\mathrm{SD}
$$

where $h_{k}(t)$ is the sifting result in the $k$ th iteration, and SD is standard deviation, typically set between 0.2 and 0.3 .

The EMD extracts the next IMF (see Fig. 4) by applying the aforementioned procedure to the residue

$$
r_{1}(t)=x(t)-c_{1}(t)
$$

where $C_{1}(t)$ denotes the first IMF. This process is repeated until the last residue $r_{n}(t)$ has at most one local extreme.

The first IMF component from the data contains the highest oscillation frequencies found in the original data $x(t)$.

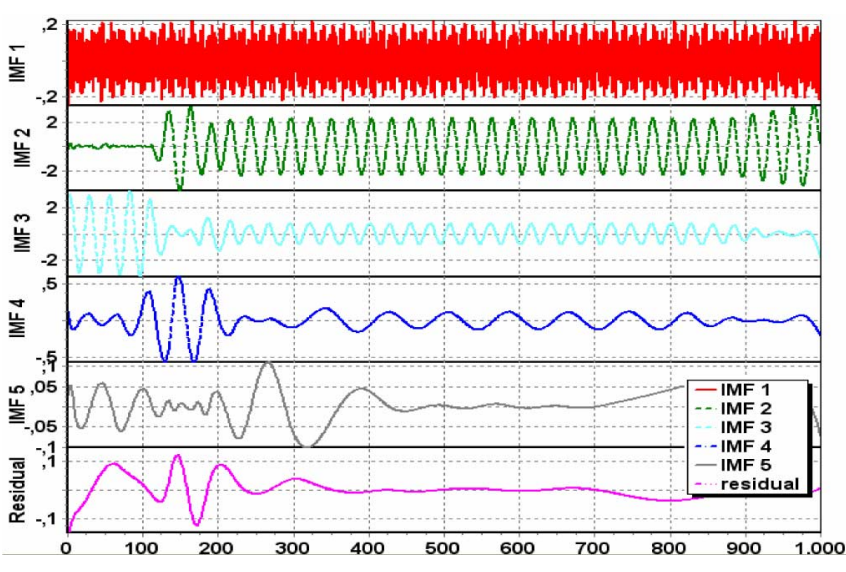

Fig. 4. IMF for a healthy PMSM. Simulations result at $1500 \mathrm{r} / \mathrm{min}$.

\section{B. Hilbert-Huang Transform}

Once the IMFs have been obtained by means of the EMD method, the Hilbert transform is performed to each IMF component as follows:

$$
H\left[c_{i}(t)\right]=\frac{1}{\pi} \int_{-\infty}^{+\infty} \frac{c_{i}(\tau)}{t-\tau} d \tau
$$

which means that $c_{i}(t)$ and $H\left[c_{i}(t)\right]$ form a complex conjugate pair, so that an analytic signal $z_{i}$ is defined as

$$
z_{i}(t)=a_{i}(t) e^{j \omega_{i}(t)} .
$$

To transform this temporal-space data to time-frequency space, the Hilbert transform is performed on each IMF component obtained by means of the EMD method as

$$
\begin{aligned}
a_{i}(t) & =\sqrt{c_{i}^{2}+H^{2}\left[c_{i}(t)\right]} \\
\theta_{i} & =\arctan \left(\frac{H^{2}\left[c_{i}(t)\right]}{c_{i}(t)}\right) .
\end{aligned}
$$

In this way, the instantaneous frequency $\omega_{i}(t)$ is given by

$$
\omega_{i}=\frac{d \theta_{i}(t)}{d t}
$$

After performing the Hilbert transform on each IMF component, we can express the data in the following form:

$$
x(t)=\operatorname{Re} \sum_{i=1}^{n} a_{i}(t) \exp \left(j \int_{-\infty}^{t} \omega_{i}(t) d t\right)
$$

where $\operatorname{Re}\{\cdot\}$ denotes the real part of a complex quantity.

Equation (5) enables to represent the amplitude and the instantaneous frequency in a 3-D figure, in which the amplitude is the length in the time-frequency plane. This time-frequency distribution is designed as the Hilbert-Huang spectrum $H(\omega, t)$ at will as

$$
H(\omega, t)=\operatorname{Re} \sum_{i=1}^{n} a_{i}(t) \exp \left(j \int_{-\infty}^{t} \omega_{i}(t) d t\right) .
$$




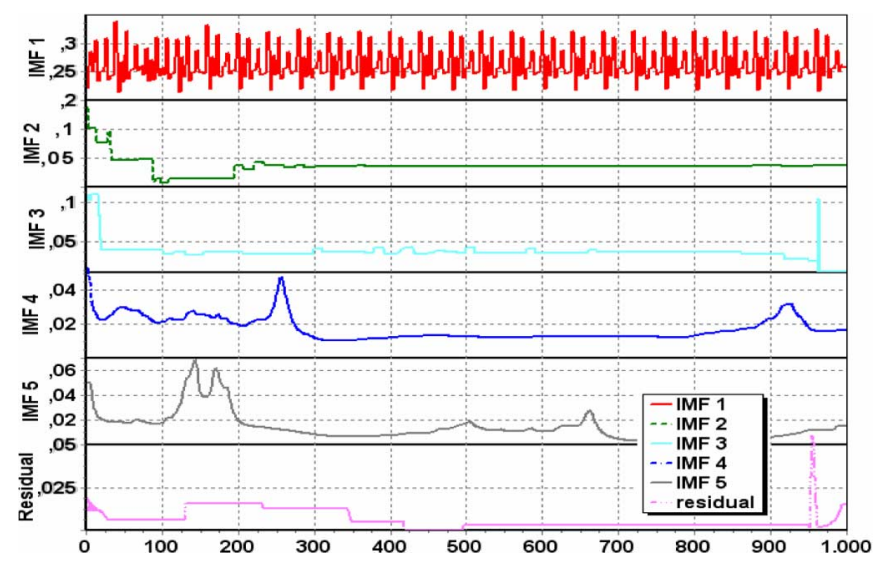

Fig. 5. Instantaneous frequency for a healthy PMSM. Simulations result at $1500 \mathrm{r} / \mathrm{min}$

With the Hilbert-Huang defined, the marginal spectrum $h(\omega)$ can be defined as

$$
h=\int_{0}^{T} H_{i}(\omega, t) d t
$$

By this way, the local marginal $h_{i}(\omega)$ spectrum offers a measure of the local amplitude contribution from the frequency that we are interested in.

Then, the marginal spectrum is computed as [14] follows.

1) To calculate the envelope signal $y(t)$ applying Hilbert transform to the current signal $x(t)$ :

$$
y(t)=\sqrt{x^{2}+H^{2}[x(t)]} .
$$

2) To decompose the envelope signal $y(t)$ using EMD and to obtain IMFs.

3) To select the interested IMF component $c_{i}(t)$ according to the objective of fault diagnosis.

4) To calculate the marginal spectrum $h_{i}(\omega)$ according to (11).

5) To analyze the marginal spectrum of selected $c_{i}(t)$ component and draw a diagnostic conclusion.

Following this procedure, the amplitude and instantaneous frequency for every IMF at every time step is computed, as shows in Fig. 5. This result can be projected on the timefrequency-energy space, with energy defined as the amplitude squared [24].

Summarizing, the HHT algorithms accurately analyze physical signals via the following steps.

1) Instantaneous frequencies are calculated based on the EMD method when IMFs are generated for complex data.

2) A Hilbert transform converts the local energy and instantaneous frequency derived from the IMFs to a full-energyfrequency-time distribution of the data.

3) The physical signal is filtered by reconstruction from selected IMFs.

4) A curve can be fitted to the filtered signal.

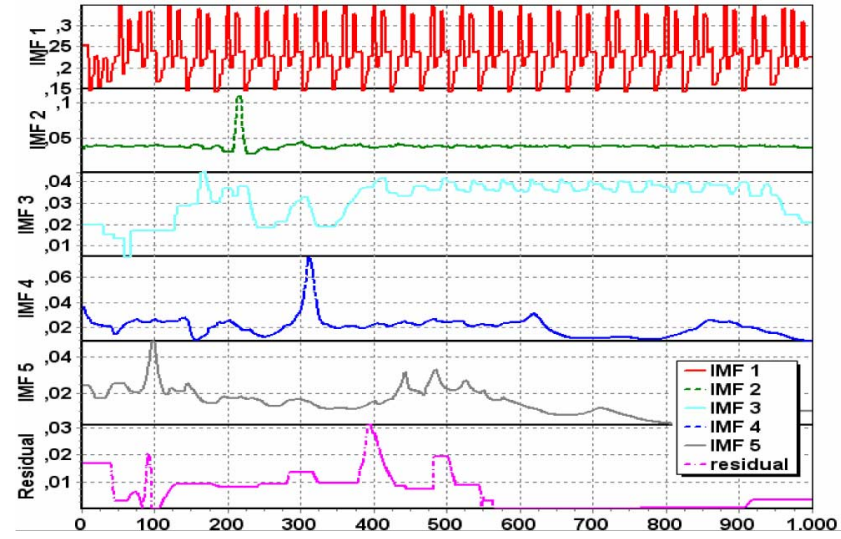

Fig. 6. Instantaneous frequency for a PMSM with 50\% demagnetization. Simulation result at $1500 \mathrm{r} / \mathrm{min}$.

\section{EXPERIMENTAL RESULTS}

The motors under analysis have been a PMSM of $6000 \mathrm{r} / \mathrm{min}$ nominal speed, $2.3 \mathrm{~N} \cdot \mathrm{m}$ nominal torque, and three poles pair. The demagnetization analysis for PMSM [1], [12] has been carried out by simulation and experimental tests for 6000, 3000, and $1500 \mathrm{r} / \mathrm{min}$ at nominal current, and faults of $75 \%$ and $50 \%$ of demagnetization. Additionally, speed variations of $500 \mathrm{r} / \mathrm{min}$ around the operational speed have been introduced through the control.

The EMD algorithm and HHT were implemented by means of software HTT Data Processing System (HHT-DPS) [25] from the National Aeronautics and Space Administration (NASA) Institution. The analysis of the current is carried out mainly for low speeds, i.e., for the cases where the classic methods of fault detection does not have kind characteristic.

The demagnetization is analyzed under different speed conditions. The stator current is divided into five IMF and HHT is calculated. The results of demagnetization machine are compared with those obtained from healthy machines.

\section{A. Steady-State Conditions: Constant Speed and Nominal Torque}

The IMF are calculated from the stator current at speeds of 6000,3000 , and $1500 \mathrm{r} / \mathrm{min}$ with nominal torque. The difference in magnitude between healthy and demagnetized machine can be shown with the instantaneous frequency in the IMF 1 and 2 in Fig. 6. The IMF 2 contains the stator-current main frequency and the others correspond to high or low frequencies with regard to the main frequency. In a similar way to simulations, experimental results in Figs. 7 and 8 show the changes in the instantaneous frequency at $1500 \mathrm{r} / \mathrm{min}$.

In the figures, IMF1, and specially IMF2, clearly show the fault for the motor damaged. As IMF allows to isolate frequency ranges in the spectra, they can be used to wrap up the frequencies that are around those of interest, and separate those that are not necessary. For instance, if one IMF isolates the stator-current main frequency, the other IMFs could be analyzed more easily to increase the precision in the failure detection, i.e., the analysis can be concentrated in specify frequency ranges. By this way, 


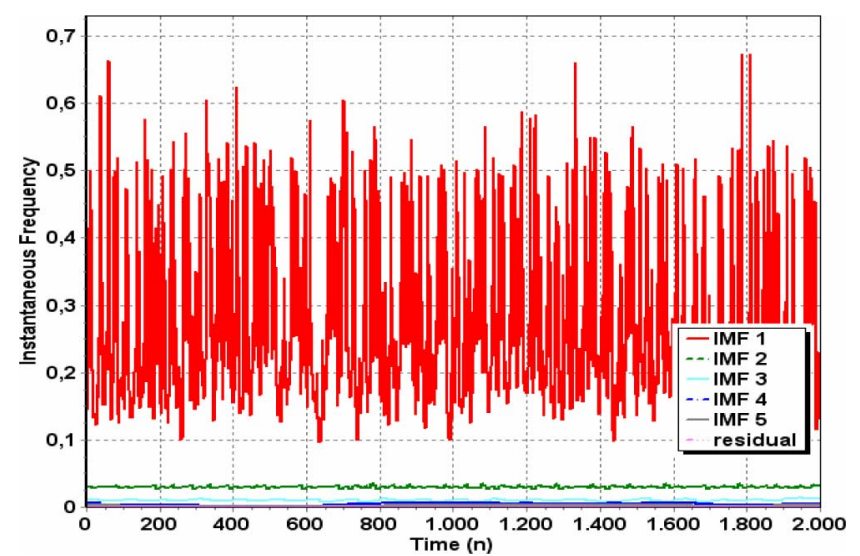

Fig. 7. Instantaneous frequency for a healthy PMSM. Experimental result at $1500 \mathrm{r} / \mathrm{min}$.

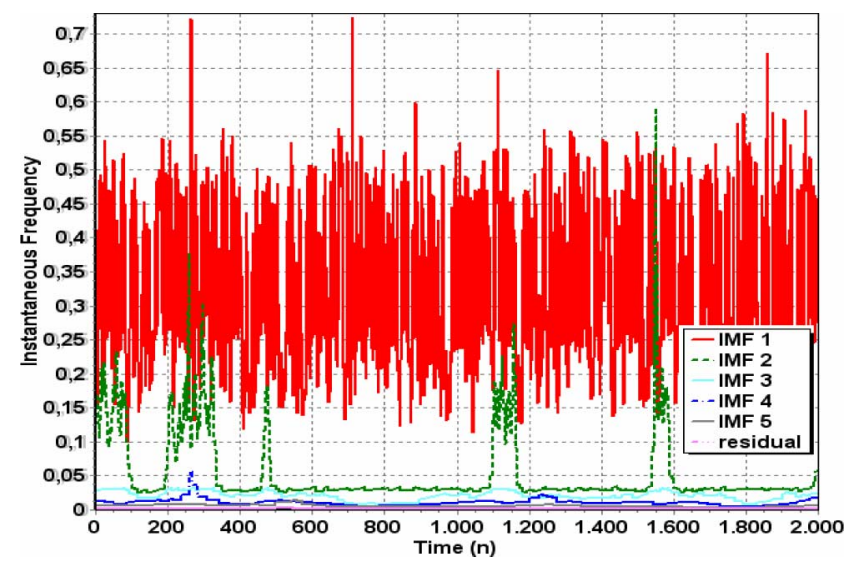

Fig. 8. Instantaneous frequency for a PMSM with $50 \%$ demagnetization. Experimental result at $1500 \mathrm{r} / \mathrm{min}$.

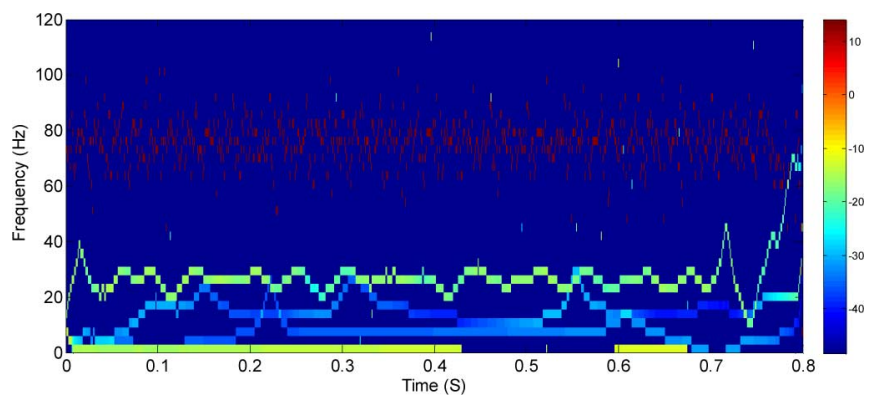

Fig. 9. HHT for a healthy PMSM. Experimental result at 1500 r/min.

the method can be extended for detection of other electric and mechanics fault.

The Figs. 9 and 10 show higher values in HHT for demagnetization machine at $1500 \mathrm{r} / \mathrm{min}$. Besides, the dynamic character of the current signal is indicated visually by means of the magnitude changes around the main frequency of $75 \mathrm{~Hz}$, the system is working at a constant torque and speed.

In Fig. 11, the instantaneous frequencies at $6000 \mathrm{r} / \mathrm{min}$ is shown. The values of IMF 1 and 2 show once again the demagnetization of the machine. The aforementioned comments are easy to understand taking into account that the demagnetiza-

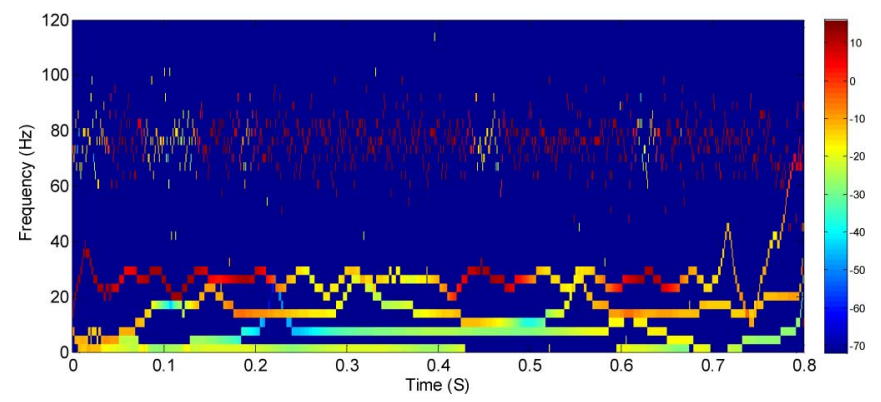

Fig. 10. HHT for a PMSM with $75 \%$ demagnetization. Experimental result at $1500 \mathrm{r} / \mathrm{min}$

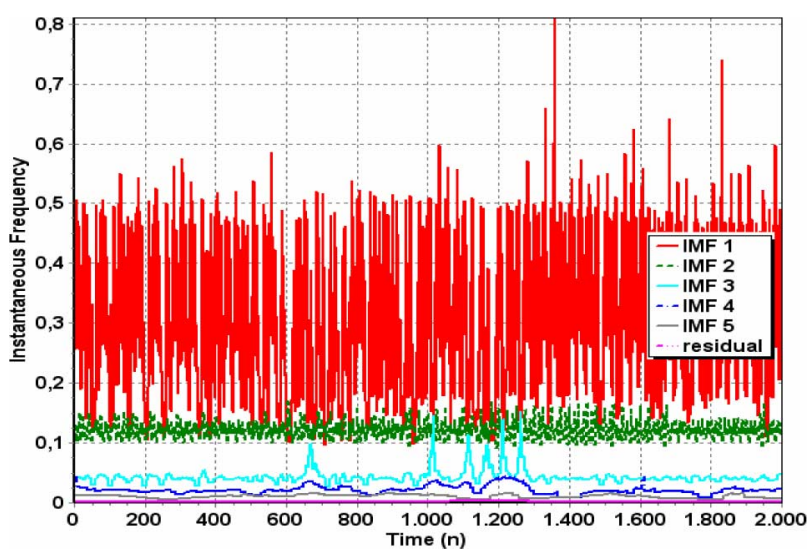

Fig. 11. Instantaneous frequency for a PMSM with 50\% demagnetization. Simulations result at $6000 \mathrm{r} / \mathrm{min}$.

tion has a great influence in the stator current, and the magnetic density induction is proportional to the speed

\section{B. Variable Conditions: Speed Change at Nominal Torque}

The PMSM usually operates to different speeds and torque conditions. Under these dynamic conditions, the demagnetization detection can be carried out by means of analysis of time-frequency of the stator current. As it could be seen in the previous section, the proposed method is able to perform fault detection at a medium speed, constant conditions.

Next, it is demonstrated that it is also useful for medium and high speeds under variable conditions. Figs. 12 and 13 depict the instantaneous frequency for healthy and demagnetized machine when the speed changes from 1500 to $1000 \mathrm{r} / \mathrm{min}$. The IMF 1 shows the speed change, while IMF 2 is centered on the statorcurrent main frequency.

Fig. 14 shows the instantaneous frequency for a demagnetized machine when a speed change form 6000 to $5500 \mathrm{r} / \mathrm{min}$ occurs.

Figs. 15 and 16 show the resulting HHT. The differences between healthy and demagnetized machines are evident, especially at the beginning of the speed change. The use of the HHT introduces better precision for the fault detection than others signal processing techniques. The HHT also shows the condition change and can be used to implement supervision systems.

Fig 17 shows instantaneous frequencies of IMFs for a damaged machine with a demagnetization fault. The results are experimental for a speed change from 1500 to $1000 \mathrm{r} / \mathrm{min}$. There 


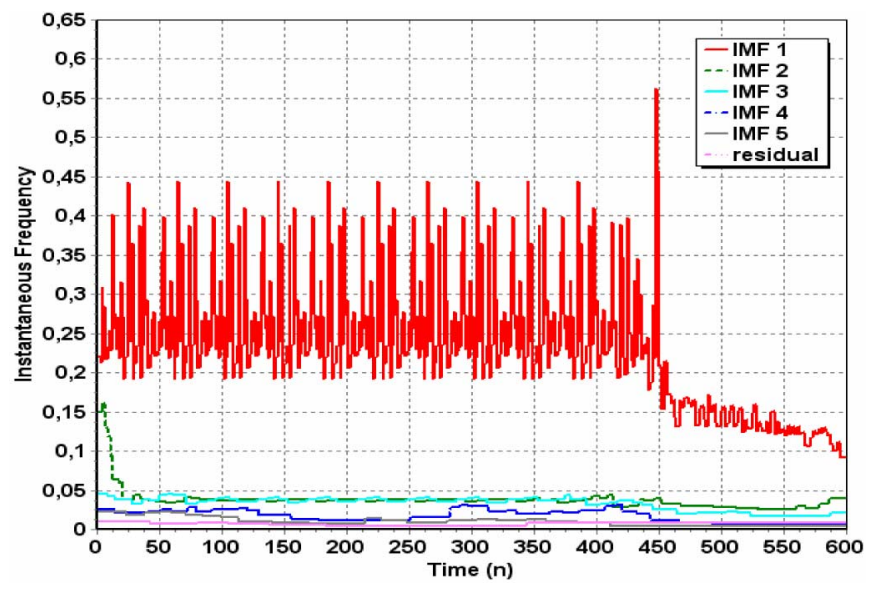

Fig. 12. Instantaneous frequency for a healthy PMSM. Speed change from 1500 to $1000 \mathrm{r} / \mathrm{min}$. Simulations result.

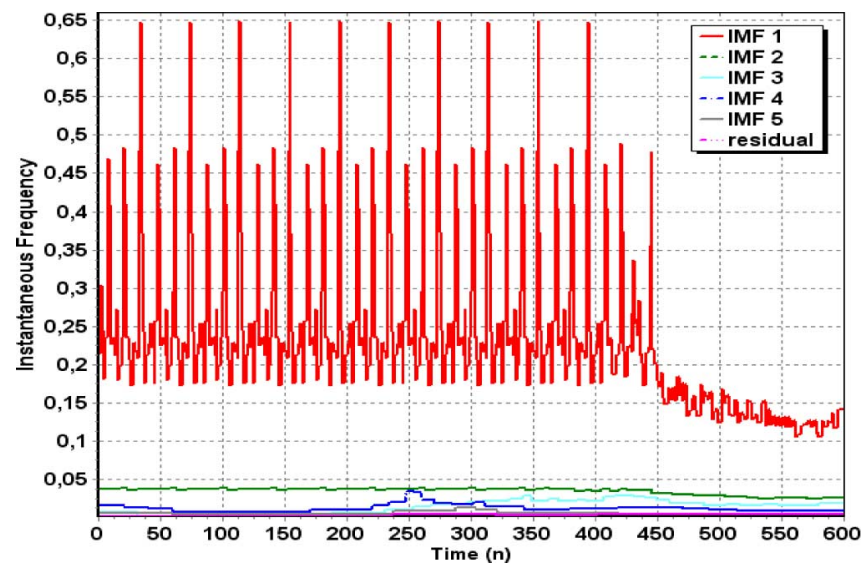

Fig. 13. Instantaneous frequency for a PMSM with $50 \%$ demagnetization. Speed change from 1500 to $1000 \mathrm{r} / \mathrm{min}$. Simulations result.

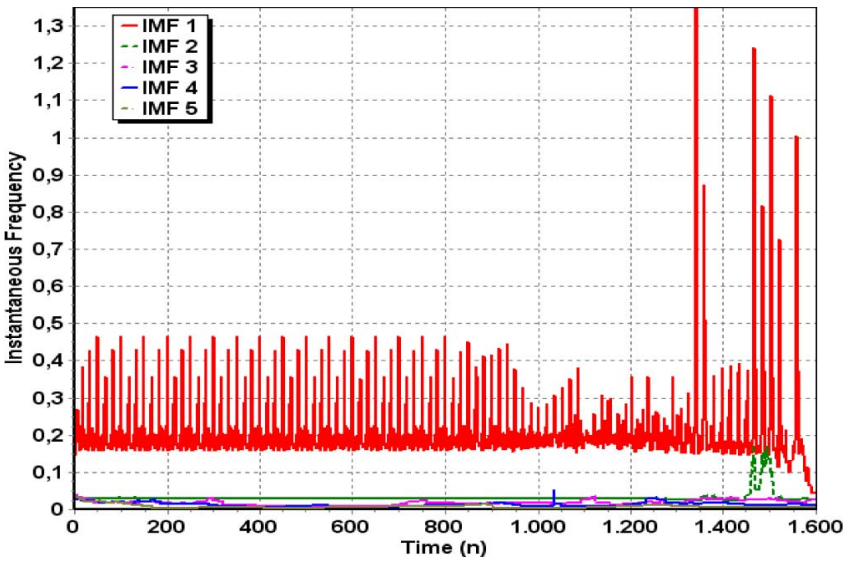

Fig. 14. Instantaneous frequency for a PMSM with $50 \%$ demagnetization. Speed change from 6000 to $5500 \mathrm{r} / \mathrm{min}$. Simulations result.

is a correlation with the simulation results, which probes the viability of this method for fault detection in PMSM.

The calculation of the IMFs depends on the signal form, the number of the samples, and the current main frequency. Therefore, it has to be carefully defined, especially when the HHT analysis should be executed.

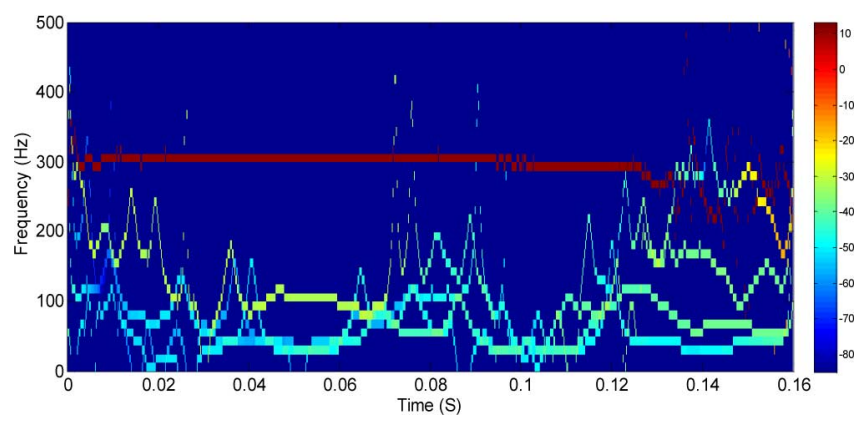

Fig. 15. HHT for a healthy PMSM. Speed change from 6000 to $5500 \mathrm{r} / \mathrm{min}$ Simulations result.

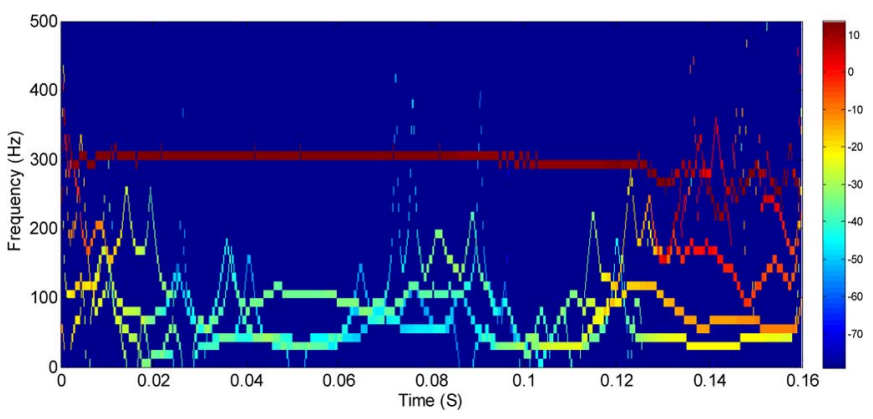

Fig. 16. HHT for a PMSM with 50\% demagnetization. Speed change from 6000 to $5500 \mathrm{r} / \mathrm{min}$. Simulations result.

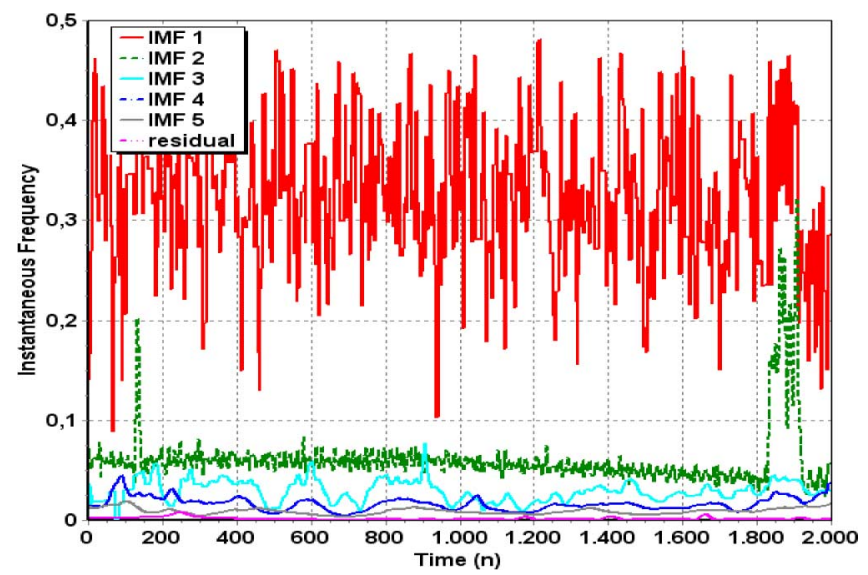

Fig. 17. Instantaneous frequency for a PMSM with $50 \%$ demagnetization Speed change from 1500 to $1000 \mathrm{r} / \mathrm{min}$. Experimental result.

The HHT allows to eliminate the undesired frequencies and concentrates the information in some IMFs, exactly those of interest for the detection of the fault frequencies.

\section{CONCLUSION}

Simulation results for a PMSM driven by a vector control have indicated that faulty harmonics are visible in the current spectrum, and FFT allows detecting demagnetization by analyzing the amplitude of first and fifth harmonics, especially for high speeds. However, fault detection with FFT is not clear at low speed, and also, it is not possible to apply FFT without steady-state operation, i.e., when there are speed and torque changes. 
Therefore, a novel signal processing technique named HTT is presented, this methodology can overcome the aforesaid drawbacks since it allows to analyze the stator current obtained from experimental data for both stable state and dynamic conditions with speed changes at high, medium, and low velocities.

The experimental results show that this method can effectively diagnose the demagnetization failure, thus obtaining an increase in the spectral resolution and also reliability compared with the motor current signature analysis, based on the FFT.

Moreover, the HHT algorithm is simple and easy to implement in a system for supervision, fault detection, and failure diagnostic by means of digital signal processor (DSP) with adequate development tools.

The application of HHT not only allows to detect the failure, but also to know the condition of operation of the machine and its changes throughout the time, as well as its trend.

\section{REFERENCES}

[1] J. Rosero, J. Cusido, A. Garcia, J. A. Ortega, and L. Romeral, "Broken bearings and eccentricity fault detection for a permanent magnet synchronous motor," in Proc. 32nd Annu. Conf. IEEE Ind. Electron. Soc., Paris, France, 2006, pp. 964-969.

[2] J. A. Rosero, J. A. Ortega, E. Aldabas, and L. Romeral, "Moving towards a more electric aircraft," IEEE Aerosp. Electron. Syst. Mag., vol. 22, no. 3, pp. 3-9, Mar. 2007.

[3] W. le Roux, R. G. Harley, and T. G. Habetler, "Detecting rotor faults in low power permanent magnet synchronous machines," IEEE Trans. Power Electron., vol. 22, no. 1, pp. 322-328, Jan. 2007.

[4] M. A. Awadallah and M. M. Morcos, "Automatic diagnosis and location of open-switch fault in brushless DC motor drives using wavelets and neurofuzzy systems," IEEE Trans. Energy Convers., vol. 21, no. 1, pp. 104-111, Mar. 2006

[5] G. Bossio, C. D. Angelo, J. Solsona, G. O. García, and M. I. Valla, "Application of an additional excitation in inverter-fed induction motors for air-gap eccentricity diagnosis," IEEE Trans. Energy Convers., vol. 21, no. 4, pp. 104-111, Dec. 2006.

[6] B. Mirafzal, R. J. Povinelli, and N. A. O. Demerdash, "Interturn fault diagnosis in induction motors using the pendulous oscillation phenomenon," IEEE Trans. Energy Convers., vol. 21, no. 4, pp. 871-882, Dec. 2006.

[7] S. Rajagopalan, J. M. Aller, J. A. Restrepo, T. G. Habetler, and R. G. Harley, "Analytic-wavelet-ridge-based detection of dynamic eccentricity in brushless direct current (BLDC) motors functioning under dynamic operating conditions," IEEE Trans. Ind. Electron., vol. 54, no. 3, pp. 1410 1419, Jun. 2007.

[8] M. A. S. K. Khan, T. S. Radwan, and M. A. Rahman, "Real-time implementation of wavelet packet transform-based diagnosis and protection of three-phase induction motors," IEEE Trans. Energy Convers., vol. 22, no. 3, pp. 647-655, Sep. 2007.

[9] I. P. Tsoumas, G. Georgoulas, E. D. Mitronikas, and A. N. Safacas, "Asynchronous machine rotor fault diagnosis technique using complex wavelets," IEEE Trans. Energy Convers., vol. 23, no. 2, pp. 444-459, Jun. 2008.
[10] G.-D. Andreescu, C. I. Pitic, F. Blaabjerg, and I. Boldea, "Combined flux observer with signal injection enhancement for wide speed range sensorless direct torque control of IPMSM drives," IEEE Trans. Energy Convers., vol. 23, no. 2, pp. 393-402, Jun. 2008.

[11] A. A. Arkadan and B. W. Kielgas, "The coupled problem in switched reluctance motor drive systems during fault conditions," IEEE Trans. Magn., vol. 30, no. 5, pp. 3256-3259, Sep. 1994.

[12] J. Rosero, J. Cusido, A. Garcia, J. A. Ortega, and L. Romeral, "Study on the permanent magnet demagnetization fault in permanent magnet synchronous machines," in Proc. 32nd Annu. Conf. IEEE Ind. Electron. Soc., Paris, France, 2006, pp. 879-884.

[13] J. Cusido, J. A. Rosero, J. A. Ortega, A. Garcia, and L. Romeral, "Induction motor fault detection by using wavelet decomposition on dq0 components," in Proc. IEEE Int. Symp. Ind. Electron., 2006, vol. 3, pp. 24062411.

[14] Z. Yuping, "Hilbert-Huang transform and marginal spectrum for detection of bearing localized defects," in Proc. 6th World Congr. Intell. Control Autom. (WCICA 2006), Dalian, China, pp. 5457-5461.

[15] J. Rosero, J. Cusido, A. Garcia, J. A. Ortega, and L. Romeral, "Broken bearings fault detection for a permanent magnet synchronous motor under non-constant working conditions by means of a joint time frequency analysis," in Proc. IEEE Int. Symp. Ind. Electron. (ISIE 2007), Vigo, Spain, 2007, pp. 3415-3419.

[16] R. Yan and R. X. Gao, "Hilbert-Huang transform-based vibration signal analysis for machine health monitoring," IEEE Trans. Instrum. Meas., vol. 55, no. 6, pp. 2320-2329, Dec. 2006.

[17] R. Yan and R. X. Gao, "A tour of the Hilbert-Huang transform: An empirical tool for signal analysis," IEEE Trans. Instrum. Meas., vol. 10, no. 5, pp. 40-45, Oct. 2007.

[18] R. Yan and R. X. Gao, "Transient signal analysis based on Hilbert-Huang transform," in Proc. IEEE Instrum. Meas. Technol. Conf., May 2005, pp. $1198-1202$.

[19] CEDRAT Group. (2005). CAD package for electromagnetic and thermal analysis using finite elements, Useŕs guide. Flux 2D. CEDRAT Group, Meylan, France [Online]. Available: http://www.cedrat.com

[20] J. F. Gieras and M. Wing, Permanent Magnet Motor Technology: Design and Applications, 2nd ed. New York: Marcel Dekker, 2002.

[21] S. Nandi, H. A. Toliyat, and X. Li, "Condition monitoring and fault diagnosis of electrical motors-A review," IEEE Trans. Energy Convers., vol. 20, no. 4, pp. 719-729, Dec. 2005.

[22] B. Liu, S. Riemenschneider, and Y. Xu, "Gearbox fault diagnosis using empirical mode decomposition and Hilbert spectrum," Mech. Syst. Signal Process., vol. 20, pp. 718-734, 2006.

[23] N. E. Huang, Z. Shen, S. R. Long, M. C. Wu, H. H. Shih, Q. Zheng, N. C. Yen, C.C. Tung, and H. H. Liu, "The empirical mode decomposition and the Hilbert spectrum for nonlinear and non-stationary time series analysis," Proc. R. Soc. A, Math., Phys. Eng. Sci., vol. 454, pp. 903-995, 1998.

[24] E. H. Norden, C. W. Man-Li, R. L. Steven, S. P. S. Samuel, Q. Wendong, G. Per, and L. F. Kuang, "A confidence limit for the empirical mode decomposition and Hilbert spectral analysis," Proc. R. Soc. A, Math., Phys. Eng. Sci., vol. 459, pp. 2317-2345, 2003.

[25] N. Huang, "Software Hilbert-Huang Transform Data Processing System (HHT-DPS)," version 1.4, NASA Goddard Space Flight Center (GSFC), Greenbelt, MD, 2003-2004.

Authors' photographs and biographies not available at the time of publication. 